16 | 2013

NOVECENTO... E DINTORNI

« On ne naît pas... on le devient "

\title{
Armanda Guiducci e le sfide dell'identità
}

Armanda Guiducci and the Challenges of Identity

Francesca Parmeggiani

CpenEdition

Journals

Edizione digitale

URL: http://journals.openedition.org/cei/1273

DOI: $10.4000 /$ cei. 1273

ISSN: 2260-779X

Editore

UGA Éditions/Université Grenoble Alpes

Edizione cartacea

Data di pubblicazione: 30 juin 2013

Paginazione: 271-280

ISBN: 978-2-84310-245-5

ISSN: $1770-9571$

Notizia bibliografica digitale

Francesca Parmeggiani, «Armanda Guiducci e le sfide dell'identità», Cahiers d'études italiennes [Online], 16 | 2013, online dal 15 décembre 2014, consultato il 27 mars 2021. URL: http:// journals.openedition.org/cei/1273 ; DOI: https://doi.org/10.4000/cei.1273 


\title{
ARMANDA GUIDUCCI E LE SFIDE DELL'IDENTITÀ ${ }^{1}$
}

\author{
Francesca Parmeggiani \\ Fordham University
}

La carriera intellettuale di Armanda Guiducci, napoletana di nascita milanese d'adozione, presenta un'evoluzione significativa. Laureata in filosofia, co-fondatrice della rivista "Ragionamenti», di cui fu anche direttrice, e studiosa di Pavese, dopo essersi affermata come critica letteraria e culturale marxista Guiducci aderisce alla causa femminista negli anni Settanta. Da questo momento darà voce alle donne raccontandone l'esperienza storica di sofferenza e protesta individuale e collettiva e, così facendo, lascerà in eredità alle generazioni future di donne e uomini un modello importante di attivismo politico e culturale.

Nei suoi scritti dagli anni Settanta in poi, Guiducci tratta della lotta che le donne hanno condotto per esprimersi, emanciparsi e liberarsi; demistifica i modi in cui il potere politico, socio-economico e culturale ha controllato o marginalizzato per secoli la presenza femminile negando al femminile valore storico e creatività artistica; infine, recupera alla memoria collettiva la presenza attiva delle donne come agenti di storia. Non rinuncia tuttavia alla sua originaria collocazione politica né dismette la veste critica degli inizi, di cui sono documento La domenica della rivoluzione (196I) e gli articoli raccolti in Dallo zdanovismo allo strutturalismo (1967), mentre la sua opera, riletta nella sua interezza, mette in evidenza l'impegno formale dell'autrice, che si cimenta con vari generi di scrittura creativa e saggistica - dai versi di Poesie per un uomo (1965) e A colpi di silenzio (1982-1989) alla narrazione (auto)biografica di La mela e il serpente. Autoanalisi di una donna (1974) e Virginia e l'angelo (199I) o di finzione in Due donne da buttare (1976) e A testa in giù (1984), dai libri di viaggio come All'ombra

I. Testo riveduto della relazione presentata al convegno internazionale "On ne nầt pas... on le devient». I gender studies e il caso italiano, dagli anni Settanta a oggi, tra liberazione sessuale e nuovi tabù tenutosi presso l'Université Stendhal-Grenoble 3 il 25-26 novembre 20 Io. 
di Kali (1979) e Il grande Sepik (1992) a quelli di storia come Perdute nella storia (1989) e Medioevo inquieto (1990), dagli studi sociologici di La donna non è gente (1977) e Donna e serva (1983) alle traduzioni o introduzioni all'opera di John Donne, Katherine Mansfield, Virginia Woolf e altri ${ }^{2}$. Nella varietà di testi e generi di scrittura che Guiducci utilizza possiamo apprezzare il coinvolgimento personale dell'autrice che mette in gioco se stessa in quanto fruitrice, mediatrice e produttrice di saperi, culture ed esperienze nel contesto delle tensioni politico-ideologiche, precipitate dalla Guerra Fredda, in una società in cui i mezzi di comunicazione di massa incrementano e velocizzano la circolazione di idee e informazione, e le differenze economiche si accentuano. La scrittura poetica, narrativa e saggistica di Guiducci, infine, si offre a noi lettori come occasione di coscienza e conoscenza, come luogo dell'espressione della soggettività e intersoggettività (non solo femminile); per l'autrice essa è pratica identitaria per eccellenza in quanto la conduce al riconoscimento di sé attraverso l'altro, nell'altro e come altro.

Entriamo perciò nell'opera di Guiducci e leggiamola come percorso emblematico, sia pure non esaustivo, della storia del pensiero femminista e del discorso sulle e delle donne in Italia dal secondo dopoguerra alle soglie del Duemila e come testimonianza originale dell'articolarsi dell'identità femminile nel secondo Novecento, delle sfide pubbliche e private, personali e politiche, sociali e culturali che tale pensiero e tale articolazione hanno proposto e tuttora propongono.

Le parole di Simone De Beauvoir poste a titolo di questo colloquio ritornano in alcuni versi della nostra scrittrice. In Il mondo aiuta l'uomo Guiducci contrappone la difficile e sofferta condizione femminile all'avvantaggiata esistenza maschile e scrive:

Diventare donna è un nascere per strappi

reiterati, per lacerazioni

là, ai margini,

dove l'erba dirada. ${ }^{3}$

2. Nonostante Armanda Guiducci sia spesso ricordata come autrice originale e influente per i suoi saggi di analisi della condizione femminile e di storia delle donne, sono pochi i lavori critici a lei interamente dedicati. Per una introduzione alla sua opera e una valutazione preliminare di alcuni aspetti della sua scrittura rimando a due saggi di Fiora Bassanese, Armanda Guiducci (1923-1992), in Italian Women Writers: A Bio-Bibliographical Sourcebook, a cura di Rinaldina Russell, Westport (CT), Greenwood Press, 1994, pp. 179-188, e Armanda Guiducci's Disposable Women, in Contemporary Women Writers in Italy: A Modern Renaissance, a cura di Santo Aricò, Amherst (MA), University of Massachusetts Press, 1990, pp. I53-I69, e a Francesca Parmeggiani, For a Politics of the Gendered Self: Armanda Guiduccis Feminist Practices, «Italian Culture», 24-25, 2006-2007, pp. 63-89, in cui esamino soprattutto la poesia di Guiducci e il rapporto tra linguaggio poetico e forma narrativa in relazione alla sua produzione di finzione e saggistica degli anni Settanta e dei primi anni Ottanta.

3. Armanda Guiducci, Il mondo aiuta l'uomo, in A colpi di silenzio, Milano, Lanfranchi, I990, p. I5I, vv. 6-9. 
La poetessa avvicina i verbi «diventare» e «nascere» associandoli attraverso «essere» nello stesso verso. Tale compresenza non nega una gerarchia: il soggetto del predicato è, dopotutto, «diventare», ma «nascere», sostantivizzato, qualifica il divenire. La struttura sintattica sottolinea l'ambiguità dell'assunto: «diventare», «essere», «nascere» sono posti in relazione; è anzi «essere» che pone in relazione "diventare» e «nascere», stabilendo un'identità tra i due termini. Il verso enfatizza l'idea di un processo, di un divenire. Questo divenire, tuttavia, si costituisce in unità, consiste e dunque permane in atti di nascita. Si tratta di un divenire per traumi, che lasciano ferite $^{4}$, ma che testimoniano gesti di consapevolezza, di affermazione di sé, di prese di parola - anch'essi traumi per le donne che vivono la lotta entro e contro il patriarcato - spesso ai margini ma non al di fuori della storia. "[D]ove l'erba dirada», osserva Guiducci, ma sempre più frequentemente al centro.

Il percorso della scrittrice verso tale consapevolezza non è né facile né ovvio. Comincia con i versi di Poesie per un uomo, in cui l'«io» lirico, una donna, dà voce al proprio desiderio e al desiderio di parlare d'amore a un uomo come un uomo parla d'amore a una donna. Il soggetto femminile si scopre e si percepisce come tale in rapporto a un "tu», di sesso maschile, che di fatto rimane distante e impenetrabile. La poesia esprime allora una differenza radicale e irrisolvibile e una distanza che solo l'atto d'amore riesce a colmare per un istante. Nella raccolta poetica successiva, dal titolo A colpi di silenzio, l'esperienza d'amore rimane centrale, ma Guiducci la racconta all'interno di una cornice propriamente femminista. In versi che parlano ancora di amore, desiderio e differenza, che parlano sempre più del corpo - un corpo anche invecchiato, non più fertile ma ancora desiderante ${ }^{5}$ - della maternità, della conoscenza di sé, delle esperienze diverse delle donne e della loro storia condivisa, dei rapporti tra di loro che attraversano il tempo, Guiducci denuncia il modo in cui le donne vengono percepite, raccontate o immaginate e celebra come esse si vedono e si raccontano:

Gli uomini non perdonano a una donna

[...]

Loro, dunque, non ti hanno perdonata.

4. Altrove nella raccolta Guiducci parlerà di «uomini-coltello» e le lacerazioni saranno ferite d'amore, un amore che "sventra», "tradisce», «divora» e «sputa», che obbedisce alle «leggi del profitto, / del potere, e della sopraffazione» (Sotto una stella impura, in A colpi di silenzio, cit., p. I33, vv. 8-I4).

5. "Mentre nel sangue vivo / un sangue morto/ ora disegna un ciclo / che si serra ad anello, / protende alte braccia il desiderio, / la speranza pulsa, / il corpo vibra» (Armanda Guiducci, Sunset Boulevard, in A colpi di silenzio, cit., p. I32, vv. 5-II). 
Ti amiamo noi, come un simbolo di noi

che patiamo gli oltraggi del tentare,

oltre l'arte della soppressione,

un senso all'esistenza femminile:

noi, in questa traslata follìa,

ti amiamo di un simbolico amore. ${ }^{6}$

Tra la prima e questa seconda raccolta poetica, Guiducci pubblica il suo testo forse più famoso e tradotto, cioè La mela e il serpente. In esso Guiducci articola una scrittura non solo affine ora al diario psicoanalitico che registra sogni, ricordi e impressioni, ora al racconto autobiografico che organizza tempo ed esperienze dall'infanzia alla maturità, ma che esibisce anche la sensibilità analitica e l'erudizione di un saggio di antropologia socio-culturale, mentre l'«io» protagonista procede alla decostruzione del sistema di simboli e consuetudini attraverso cui la società occidentale ha condannato la donna al silenzio e alla sottomissione. Per la scrittricenarratrice la presa di coscienza di tale condizione è propedeutica al riconoscimento positivo di sé e del proprio corpo e alla generazione, auspicata alla fine, di un 'nuovo' soggetto femminile.

La scrittura poetica e autoanalitica interpretata come pratica identitaria del soggetto femminile determina dei cambiamenti profondi nella scrittura saggistica, di esplicito impegno politico, di Guiducci: la sfida personale di una donna tesa a conoscere e affermare se stessa e a denunciare il sistema simbolico del patriarcato continua, ma diviene sfida ai sistemi culturali, socio-economici e politici in cui l'identità femminile si forma e agisce. Il progetto di Guiducci, che ripropone il tema particolarmente sentito in Italia della doppia militanza e della contrapposizione tra radicalismo anti-istituzionale e anti-ideologico da una parte, e necessità di relazione con la politica istituzionale e le istituzioni della politica dall'altra, è ambizioso. L'autrice si fa portavoce e interprete della militanza politico-culturale delle donne nella storia, e mentre esplora il rapporto tra patriarcato e capitalismo recupera ma anche trasforma la propria originaria formazione intellettuale e appartenenza ideologica. Pur riconoscendo infatti che marxismo e femminismo si offrono entrambi come pratiche rivoluzionarie per attuare cambiamenti socio-economici e promuovere uguaglianza, Guiducci critica l'indifferenza del marxismo verso lo sfruttamento, la subordinazione e l'emarginazione delle donne in quanto donne: il marxismo infatti riconduce la «questione femminile» ai principi della

6. Armanda Guiducci, Silvia Plath, in A colpi di silenzio, cit., p. I48, vv. 6, I3-19. Per un'analisi approfondita dell'opera poetica di Guiducci rimando a Francesca Parmeggiani, For a Politics of the Gendered Self, cit. 
lotta di classe e della relazione dei lavoratori alla produzione del capitale; in realtà la risolve - o meglio dissolve - facendosi complice del sistema che vuole cambiare ${ }^{7}$. Allo stesso tempo, però, critica, definendolo «borghese» ed "elitario», il femminismo a lei contemporaneo, in quanto tende a parlare delle donne nelle città - condizione che lei stessa esamina con i ritratti della casalinga e della prostituta di Due donne da buttare - e a dimenticare la condizione di doppia esclusione o subalternità delle donne nelle campagne. Attraverso le testimonianze raccolte in La donna non è gente Guiducci vuole rompere il duplice silenzio che le affligge, la loro «silenziosa voce soffocata», effetto sia dell'esclusione causata dal sistema patriarcale che caratterizza la vita rurale sia dell'emarginazione prodotta dal femminismo dominante che lotta principalmente per i diritti delle donne nelle aree urbane e industrializzate ${ }^{8}$. E quando dichiara:

Il circolo vizioso della povertà economica e della subalternità femminile esclude ancora le escluse dalle stesse potenzialità di liberazione che si vanno manifestando nel mondo attuale. Queste lotte sono già un privilegio sociale, quasi un lusso da 'dominanti'. Rompere quel cerchio sarebbe il primo compito del femminismo, tagliando le radici prime dove la miseria e l'esclusione si confermano attraverso la miseria e l'esclusione stesse, lasciando intatti i poteri dominanti',

Guiducci usa di fatto il suo training critico-intellettuale per resistere (o offrire un correttivo) alla tentazione essenzialista del femminismo che, parlando della donna, si fa ideologia, estranea tanto alle necessità materiali quanto alle realtà culturali, individuali o condivise, delle donne.

Gli scritti più esplicitamente politici e socialmente impegnati di Guiducci riflettono dunque sia l'evoluzione del suo pensiero sia il rapporto dialettico che tenta di stabilire tra queste due pratiche e culture politiche, ossia il marxismo da un lato e il femminismo dall'altro. La sua voce ne risulta talvolta divisa o sdoppiata, ma l'originalità della sua scrittura è proprio nella tensione tra queste diverse esperienze di vita e di pensiero. Come ulteriore esempio, consideriamo brevemente $L a$ domenica della rivoluzione e Donna e serva, testi particolarmente rappresentativi della formazione politica di Guiducci visto che in entrambi la scrittrice si concentra su problemi di carattere sociale ed economico con atteggiamento critico sia verso il sistema capitalistico americano sia

7. La critica di Guiducci riflette il dibattito animato, non solo in Italia, tra gli anni Settanta e Ottanta sul rapporto tra marxismo e femminismo, per il quale rimando al saggio di Heidi Hartmann, The Unhappy Marriage of Marxism and Feminism: Towards a More Progressive Union, in The Second Wave: A Reader in Feminist Theory, a cura di Linda Nicholson, New York, London, Routledge, 1997, pp. 97-I22.

8. Armanda Guiducci, La donna non è gente, Milano, Rizzoli, I977, p. 17.

9. Ibid., p. I8. 
verso il modello comunista dell'Unione Sovietica. In La domenica della rivoluzione Guiducci racconta il suo viaggio in Unione Sovietica dopo la denuncia staliniana di Chruščëv e la soppressione della rivoluzione ungherese del 1956. La posizione espressa nei confronti di Stalin - manipolatore dell'indolente «anima» russa in nome in una distorta utopia marxista volta a soddisfare la propria individualistica volontà di potere - e la continua critica del modello americano ci presentano una Guiducci allineata con la posizione del PCI in quegli anni ${ }^{10}$. In Donna e serva, invece, il discorso tradizionalmente politico si apre all'esame della condizione delle donne in Italia e altrove. Guiducci affronta i temi dell'emancipazione e della liberazione femminile, della subordinazione delle donne attraverso lo sfruttamento del lavoro domestico - che definisce «obbligata servitù», gratuita, senza orari, immobile e a vita ${ }^{\mathrm{II}}$ - e del loro asservimento e confinamento attraverso il controllo della loro sessualità e della loro affettività nel matrimonio e nella maternità. Tale condizione di esclusione e «sottrazione sociale di potere» ha avuto un impatto gravissimo, sia a livello personale sia a livello sociale, sulle donne di ogni tempo ma soprattutto in epoca moderna, in quanto le ha portate alla spontanea «rinuncia alla lotta per il potere» e alla svalutazione autodistruttiva di sér ${ }^{12}$. In generale l'analisi di Guiducci rivela e colpisce polemicamente i limiti di una lettura marxista della società e della storia che rimanga indifferente alla categoria del genere, anche se risulta fortemente problematica e contraddittoria là dove, denunciati il capitalismo occidentale e il comunismo sovietico, guarda alla Cina di Mao positivamente, come a una società in cui la donna, uguale all'uomo in quanto con lui condivide ogni mansione familiare, non è più schiava del proprio ruolo domestico e materno ${ }^{13}$.

Lo sdoppiamento della voce femminile, la pluralità di esperienze che essa racconta di sé e di altre e il dialogo tra donne attraverso la storia, cui Guiducci partecipa e contribuisce, sono tematizzati anche in Virginia e l'angelo, una biografia di Virginia Woolf che invito a leggere come autobiografia di Armanda Guiducci, come racconto ulteriore del sé autorevole. Guiducci vi descrive l'educazione alto-borghese vittoriana della Woolf, il suo rapporto con il circolo di Bloomsbury, l'attivismo politico nel Regno Unito all'inizio del secolo, le relazioni familiari, sentimentali e di ami-

Io. Per una ricostruzione critica del rapporto tra $\mathrm{PCI}$ e politica sovietica si veda Paul Ginsborg, A History of Contemporary Italy: Society and Politics 1943-1988, London, Penguin, 1990, in particolare pp. 204-209 sugli eventi del 1956.

II. Armanda Guiducci, Donna e serva, Milano, Rizzoli, 1983, pp. 2I-22.

I2. Ibid., p. 75 .

13. Su questo si veda anche Francesca Parmeggiani, For a Politics of the Gendered Self, cit., p. 80. 
cizia della Woolf, il matrimonio con Leonard, la malattia, il suicidio. Allo stesso tempo il libro offre un'interessante ricostruzione della storia inglese ed europea nei primi decenni del Novecento con particolare attenzione ai temi dell'educazione femminile, della tutela del lavoro delle donne, dell'associazionismo femminile e della battaglia emancipazionista per il diritto al voto.

Guiducci, tuttavia, sconvolge l'orizzonte d'attesa del lettore in quanto non organizza il suo racconto linearmente e presentando le sue fonti in modo documentario-oggettivo. Già il titolo suggerisce un'idea di esistenza non come ordinata sequenza cronologica di eventi ed esperienze, ma come relazione e tensione tra personaggi reali (Virginia) e simbolici (l'angelo come emblema di protezione ma anche conflitto e confronto). Il polarismo del titolo entra nel testo: Virginia è Jinny $e$ Virginia.

La biografia comincia in questo modo:

Grigie ciocche cadranno dalle tempie di Virginia e, fra le guance scavate, il suo profilo assottigliato si tenderà, inquieto e pensoso, verso un'ossessiva visione di morte: una pinna emergente dal mare deserto. E ormai quasi del tutto trascorso fluirà il tempo di sabbia della sua vita, eppure là, sotto le tempie imbiancate, dove la memoria pulsa sul sangue di un'esistenza intera, là resterà l'unghiata, fresca, lacerante, segreta. ${ }^{\mathrm{I}}$

Le ultime frasi di questa citazione rimandano a un trauma, al segreto che la biografia svelerà. Subito dopo Virginia cede il palcoscenico testuale a Jinny, una bimba sensibilissima ma serena che si sta godendo le vacanze, l'aria, la luce del sole, l'odore del mare a Talland House e che gioca con il fratellastro Gerald, che la violenterà. La frammentazione dell'io che lo sdoppiamento del personaggio suggerisce in apertura di racconto rimarrà costante nel testo. Nella figurazione di Guiducci la causa di tale sdoppiamento, la ferita della violenza subìta, non si risolverà e anzi sarà la ragione per cui Virginia Woolf cancellerà il corpo e la sessualità femminile dai suoi testi, né mai riuscirà a liberarsi interamente del modello paterno — e dell'autorità maschile che esso rappresenta in una cultura tradizionalista — o di quello materno significante la sottomissione femminile in una società patriarcale. Guiducci riconoscerà tuttavia che questo limite nella scrittura di Woolf e la complessità che ne risulta impediscono che la sua opera sia riconducibile a un unico sistema interpretativo, e anzi rendono la scrittrice inglese «maestra» o «madre» alle donne del Novecento e autrice originale e innovativa nella modernità letteraria. 
Alla scissione di Jinny e Virginia Guiducci aggiunge altre voci, che talvolta proteggono, talvolta antagonizzano la protagonista, intensificando così la tensione interna al testo: la madre, Vanessa, Violet, Janet, Vita e Leonard sono gli angeli custodi della sua vita, ai quali si rivolge per ottenere conforto, protezione e conoscenza, ma sono anche demoni che impongono o suggeriscono limitazioni, norme comportamentali o addirittura il silenzio. Tra queste voci, ora sopraffatta da esse ora invece magistrale e autoritaria, è anche quella di Guiducci che sente, liricamente, come la sua protagonista, oppure la giudica severamente. Da un lato ne condivide i moti del cuore e della mente e la scrittura, attraverso cui li racconta, si addolcisce e si arricchisce di immagini. Dall'altro, invece, Guiducci critica il socialismo di Virginia ritenendolo superficiale, o denuncia la mancanza di un vero impegno politico da parte della scrittrice inglese. In questi brani, Guiducci fa sentire la sua voce di polemista, quasi priva di empatia. Assistiamo, dunque, a uno sdoppiamento nella forma del testo: il lirismo e metaforismo della Guiducci scrittrice sussiste a fianco o contro il razionalismo stilisticamente poco attraente della Guiducci saggista; la biografia diventa autobiografia, che dice tanto dell'oggetto osservato quanto del soggetto osservante. Il discorso indiretto è la tecnica più efficace di cui Guiducci si serve per organizzare il racconto; così le voci fluiscono l'una nell'altra, frammentate da flaskback e improvvisi salti nel futuro, oltre il limite temporale della biografia di Virginia Woolf e dell'esperienza dei suoi contemporanei, oltre addirittura il tempo di Guiducci, nel nostro tempo di lettrici. Continuando e perfezionando il percorso inaugurato con La mela e il serpente e con le storie di Due donne da buttare, i ritratti di La donna non è gente e il racconto $A$ testa in giù, Guiducci articola qui l'infinito movimento della lettura di sé, di una scoperta e una comprensione che coinvolgono tanto Woolf quanto la lettrice, non più perdute $\mathrm{o}$ marginalizzate nella storia.

Conoscere è esistere: l'esistenza è tanto del soggetto che conosce quanto dell'oggetto o meglio dei soggetti che vengono conosciuti, ed esistere significa diventare attraverso nuove nascite, che vogliono attraversare e oltrepassare confini culturali, temporali e geografici. L'ultima tappa dell'itinerario intellettuale ed esistenziale di Guiducci sono le testimonianze, tra letteratura di viaggio e trattato etnografico, che la scrittrice ci lascia, in All'ombra di Kali e in Il grande Sepik, dei suoi viaggi rispettivamente in India e Nepal e in Australia e Papua Nuova Guinea. Ecco la nuova sfida dell'identità femminile - in costante formazione e sempre in movimento - quella che trasforma il femminismo in esperienza e impegno transnazionali e accetta il rischio di un orientalismo dal punto di vista 
femminile, nella consapevolezza che, scrive Guiducci, «non esistono soluzioni individuali» alla crisi della civiltà occidentale, alla violenza che essa ha perpetrato nei secoli contro le donne, alla perdita della sua "capacità di contrastare la morte sulla terra con i progetti e la grandezza visionaria», e nella convinzione che "[l]e strade della liberazione sono collettive» ${ }^{15}$. Le parole di Guiducci, con cui ci congediamo, esortano a superare la tentazione dell'isolamento, della solitudine che rassicura e protegge e, in fondo, del narcisismo della differenza, a favore di un impegno comune all'azione culturale, sociale e politica, di una presenza attiva nella storia in nome di una democrazia dell'uguaglianza che è veramente tale solo se accoglie, rispetta e valorizza le differenze.

\section{Bibliografia delle opere citate}

Bassanese Fiora, Armanda Guiducci's Disposable Women, in Contemporary Women Writers in Italy: A Modern Renaissance, a cura di Santo Aricò, Amherst (MA), University of Massachusetts Press, 1990, pp. 153-I69.

-, Armanda Guiducci (1923-1992), in Italian Women Writers: A BioBibliographical Sourcebook, a cura di Rinaldina Russell, Westport (CT), Greenwood Press, 1994, pp. I79-I88.

Ginsborg Paul, A History of Contemporary Italy: Society and Politics 1943-1988, London, Penguin, I990.

Guiducci Armanda, La domenica della rivoluzione, Milano, Lerici, I96I.

—, Poesie per un uomo, Milano, Mondadori, 1965.

—, Dallo zdanovismo allo strutturalismo, Milano, Feltrinelli, 1967.

—, La mela e il serpente. Autoanalisi di una donna, Milano, Rizzoli, 1974.

—, Due donne da buttare, Milano, Rizzoli, 1976.

—, La donna non è gente, Milano, Rizzoli, 1977.

—, All'ombra di Kali, Milano, Rizzoli, 1979.

—, Donna e serva, Milano, Rizzoli, 1983.

—, A testa in giù, Milano, Rizzoli, 1984.

—, Perdute nella storia, Firenze, Sansoni, 1989.

-, A colpi di silenzio, Milano, Lanfranchi, I990.

—, Medioevo inquieto, Firenze, Sansoni, I990.

-, Virginia e l'angelo, Milano, Longanesi, I99I.

—, Il grande Sepik. Il tramonto del primitivo, Milano, Lanfranchi, 1992. 
Hartmann Heidi, The Unhappy Marriage of Marxism and Feminism: Towards a More Progressive Union, in The Second Wave: A Reader in Feminist Theory, a cura di Linda Nicholson, New York, London, Routledge, 1997, pp. 97-I22.

Parmeggiani Francesca, For a Politics of the Gendered Self: Armanda Guiducci's Feminist Practices, «Italian Culture», 24-25, 2006-2007, pp. 63-89. 